\title{
Real-World Fetal Exposure to Acne Treatments in the United States: A Retrospective Analysis from 2006 to 2015
}

\author{
Yasser Albogami $^{1,2,3}$ (D) Amir Sarayani ${ }^{1,3}$ - Juan M. Hincapie-Castillo ${ }^{1,3}$ (D) Almut G. Winterstein ${ }^{1,3}$ (D)
}

Accepted: 3 February 2021 / Published online: 8 March 2021

(c) The Author(s) 2021

\begin{abstract}
Introduction Several studies have evaluated the effects of changes in isotretinoin risk mitigation programs, but little is known about actual fetal exposure rates in the context of other acne treatments.

Objective Our objective was to quantify fetal exposure rates during the use of common acne treatments.

Methods Employing the insurance claims data of > 100,000 acne treatment users between 2006 and 2015, we created three user cohorts: (1) isotretinoin (strong teratogen/mandatory risk mitigation program), (2) doxycycline/minocycline (mild teratogen, label warning), and (3) topical clindamycin/erythromycin (no fetal risk). Fetal exposure rates overall and stratified by age were compared after adjusting for potential confounders.

Results Contraceptive use during acne treatment was $<50 \%$ in isotretinoin users and $<30 \%$ in the other study groups. Long-acting contraceptives contributed to $1 \%$ of all contraceptives used, with $90 \%$ being oral contraceptives. Isotretinoin users had 19.2 (95\% confidence interval [CI] 20.3 to 17.9) fewer fetal exposures per 1000 person-years of use compared with doxycycline/minocycline users, which in turn had 28.8 (95\% CI 31.2 to 26.3) fewer pregnancies compared with clindamycin/erythromycin users. Stratification by age showed attenuated differences in fetal exposure among acne treatment groups for teenagers.

Conclusion Fetal exposure to acne treatments varied according to levels of teratogenicity, with reduced rates among users of isotretinoin and to a lesser extent doxycycline/minocycline. Teenagers had low pregnancy rates but less pronounced differences in fetal exposure across acne treatments.
\end{abstract}

\section{Introduction}

One of the most stringent drug risk management programs imposed by regulators worldwide aims to prevent fetal exposure to isotretinoin, a potent acne treatment with serious risk for fetal malformations when used during pregnancy $[1,2]$. Since isotretinoin was approved in the USA in 1982, regulations aimed at ensuring safe use have changed several times [3]. Today, women of childbearing potential

Almut G. Winterstein

almut@ufl.edu

1 Department of Pharmaceutical Outcomes and Policy, College of Pharmacy, University of Florida, Gainesville, FL, USA

2 Department of Clinical Pharmacy, College of Pharmacy, King Saud University, Riyadh, Saudi Arabia

3 Center for Drug Evaluation and Safety, University of Florida College of Pharmacy, 1225 Center Drive, HPNP Building, Rm 3334, PO Box 100496, Gainesville, FL 32610, USA
(WCBP) planning to use isotretinoin must be registered in the iPLEDGE system for at least 30 days preceding their first prescription, during which they must take a pregnancy test and start using at least two methods of contraception (or indicate abstinence). To be eligible for a prescription refill after receiving a maximum of a 30-day supply, women must show evidence of a negative pregnancy test and continuation of contraceptive use [3].

Previous research has demonstrated that iPLEDGE has not significantly prevented fetal exposure compared with its predecessor program, which had similar requirements but relied on manual verification of negative pregnancy tests when dispensing isotretinoin [4]. Studies that have evaluated fetal exposure rates against the absence of any risk management strategy are lacking because of the long history of regulatory restrictions. Thus, iPLEDGE's overall effectiveness and the contribution of each individual risk management component within iPLEDGE are unclear [5]. In the absence of an appropriate comparison period when no risk management was in place, there are opportunities to 


\section{Key Points}

Doxycycline and minocycline users had about half the pregnancy rate of clindamycin and erythromycin users.

Pregnancy rates across acne treatment groups representing teenagers were more similar than rates among females in their twenties and thirties.

Less than half of isotretinoin users used hormonal contraceptives, and a negligible proportion used long-acting contraceptives.

evaluate rates of fetal exposure to isotretinoin in the context of exposure rates to other acne treatments used by women expected to be similar to isotretinoin users. Such an evaluation would be particularly meaningful when focused on the most prevalent age groups among users of acne treatments, i.e., teenagers and young adults, because of expected differences in risk-taking behavior, access to contraception, and desire for pregnancy [6].

Accordingly, the aim of this study was to quantify fetal exposure to acne drugs with various pregnancy prevention strategies overall and stratified by age using real-world data.

\section{Methods}

\subsection{Data Source}

We used data from the IBM ${ }^{\circledR}$ MarketScan ${ }^{\circledR}$ Commercial Database from 1 March 2006, the date of iPLEDGE approval, to 30 June 2015. MarketScan includes the enrollment and billing records of a large national sample of individuals with employer-sponsored health insurance.

\subsection{Study Population}

We considered all WCBP (age 15-44 years) with one outpatient encounter with a diagnosis of acne (International Classification of Diseases, Ninth Edition, Clinical Modification code 706.1) [7] and with at least 6 months of continuous health plan enrollment with drug coverage before the study index date, which was defined as initiation of any study drug. The initiation of the study drugs (index date) was required to be within 7 days of any acne diagnosis. We required patients to have had a diagnosis of acne within 7 days of the index date to ensure the study drugs were prescribed for acne to mitigate confounding by indication. We excluded women who had undergone hysterectomy, bilateral oophorectomy, total abdominal hysterectomy, or diagnoses of premature menopause, natural menopause, or sterility. We further excluded women who had pregnancy-related medical encounters without evidence of delivery or termination. To mitigate any cross-over effect on women's behavior because of the known risk of the other teratogen, we excluded women who used other medications with moderate or high teratogenic risk defined according to the Teratology Information System database [8]. To ensure the study population initiated the study drug for acne treatment, we also excluded women with infections that could be alternative indications during the 30 days before the index date. Finally, WCBP had to have at least 3 months of continuous enrollment after drug discontinuation or an indication for conception, whichever occurred sooner. This requirement was added to ensure that all pregnancies were captured. For instance, women who switched insurance after pregnancy may otherwise have been misclassified as not pregnant.

\subsection{Exposure Definition}

We created three exposure groups based on initiation of three groups of acne treatments: (1) isotretinoin with iPLEDGE risk evaluation and mitigation strategy (REMS), (2) oral doxycycline or minocycline with label warning for permanent dental discoloration and recommendation to avoid during pregnancy $[9,10]$, and (3) topical erythromycin or clindamycin with unknown pregnancy risk $[8,11]$. All exposure groups were allowed to use other topical acne treatments such as benzoyl peroxide. Women who initiated isotretinoin were considered exposed from the dispensing date until 30 days following the last day of supply because iPLEDGE instructions require avoidance of pregnancy for at least 30 days after the last isotretinoin dose [12]. Exposure to the other comparator drugs was assumed until the last day of supply. Patients were censored if they switched to the study comparators or if no prescription fill occurred within 45 days, thus focusing on the first treatment episode.

\subsection{Outcome Definition}

The study outcome was conception during the exposure period. To estimate conception date, we used an algorithm based on previous validation studies that identified pregnancy episodes among WCBP [13-16].

\subsection{Contraceptive Use Definition}

To evaluate the use of contraceptives during acne treatment, we considered all dosage forms of hormonal contraceptives as well as copper intrauterine devices. Contraceptive exposure was identified from pharmacy dispensing claims based on national drug codes and from outpatient encounters using procedure codes that indicated administration of contraceptives or insertion or removal of devices. For oral, patch, 
and vaginal rings, exposure time was defined as the day of dispensing until exhaustion of the provided days' supply. The effective duration of injectables was assumed to be 90 days [17]. For implants and intrauterine devices, start and end dates for contraceptive use were based on insertion and removal claims.

\subsection{Covariate Selection}

Considered potential confounders included age, geographic region, health plan type, insurance holder (whether employee, spouse, or child), and calendar year at index date as well as the following variables obtained during the 6-month pre-index period: exposure to other acne treatments (including tretinoin, adapalene, tazarotene, and benzoyl peroxide), procedures to treat acne [18] (e.g., skin peeling), history of pregnancy termination or delivery, presence of comorbidities summarized via the Charlson Comorbidity Index (CCI) [19], depression [20], and history of contraceptive use 60-180 days before treatment initiation (including hormonal contraceptives and copper intrauterine devices) $[21,22]$. This time frame was chosen to ensure no influence of planned acne treatment on contraceptive use.

\subsection{Statistical Analysis}

In each exposure group, we calculated the crude pregnancy incidence rate and the prevalence of each pregnancy outcome. Prevalence of contraceptive use during acne treatment was calculated and stratified by age groups.

Crude incidence rate ratios (IRRs) and incidence rate differences (IRDs) were estimated across exposure groups including all women and stratified by age. To adjust for potential confounders, we generated a propensity score by fitting a logistic regression model for each of the three comparisons of two exposure groups. Stabilized inverse probability of treatment weights (SIPTW) were then computed for each comparison. The SIPTW results in a weighted sample in which potential confounders that were included in the weights are balanced [23]. We then reported the IRRs and IRDs for each of the weighted comparisons. We conducted sensitivity analyses to examine the robustness of the study results. First, we ignored the requirement to continue contraception for 30 days after isotretinoin discontinuation to compare pregnancy risk during identical exposure definitions of comparison groups. Second, we solely included patients with no history of any acne treatment (naïve users).

Analyses were performed using SAS software, version 9.4 (SAS Institute, Inc.), and graphs were generated using R-package ggplot2 v2.2.1, version 3.4.2 (R Foundation for Statistical Computing) [24].

\section{Results}

Among WCBP who met the inclusion and exclusion criteria, we identified those who initiated isotretinoin $(n=84,204)$, doxycycline or minocycline (label warning) $(n=473,167)$, or erythromycin or clindamycin (no known risk) $(n=422,318)$ (Fig. 1 in the electronic supplementary material [ESM]). User groups had similar age distributions and comorbidities, but isotretinoin initiators included a larger proportion of women with a history of contraceptive use (34.1\%) compared with the label warning $(21.2 \%)$ and no-risk $(23 \%)$ cohorts (Table 1). Baseline characteristics were well balanced between exposure groups after applying SIPTW, with standardized differences below the 10\% threshold (Figs. 2-4 in the ESM).

About $49.5 \%$ of isotretinoin users had at least of 1 day of contraceptive use during treatment, compared with 25.1 and $22.9 \%$ among doxycycline/minocycline and no-risk cohorts, respectively (Table 2). Prevalence of contraceptive use was slightly lower among teenagers, with $45 \%$ among isotretinoin initiators, $20 \%$ among doxycycline/minocycline initiators, and $17 \%$ among clindamycin/erythromycin initiators. Oral hormonal contraceptives were most commonly used $(\sim 90 \%)$.

We identified a total of 5179 conceptions during followup. Livebirth delivery was the most frequent pregnancy outcome used to estimate conception date in the doxycycline/ minocycline $(61.1 \%)$ and no-risk cohorts $(66.3 \%)$, whereas conception date in the isotretinoin cohort was estimated most commonly based on abortion events (42.5\%, Fig. 1). In addition, $14.7 \%$ of conceptions in the isotretinoin cohort were identified solely from routine encounters, with pregnancy diagnosis without subsequent encounter indicating a pregnancy outcome. Label warning and no-risk cohorts had only about $11 \%$ of conceptions identified without a known pregnancy outcome.

Pregnancy rates were lowest for isotretinoin, with 5 per 1000 person-years (95\% confidence interval [CI] 4.3-5.8), followed by the label warning $(25.2 ; 95 \%$ CI 24.3-26.2) and no-risk cohorts (57.6; 95\% CI 55.3-60) (Table 3). Teenagers, the most common age group across all exposure groups, had the lowest pregnancy rates, with 1.7, 4.6, and 5.5 per 1000 WCBP-years for isotretinoin, label warning, and norisk cohorts, respectively. After applying SIPTW, the IRD of pregnancy risk during isotretinoin exposure versus exposure to doxycycline/minocycline was -19.2 (95\% CI -20.3 to - 17.9) per 1000 person-years, indicating a $77 \%$ decrease (Table 4). Compared with erythromycin or clindamycin (no known risk), isotretinoin users had 50.1 fewer pregnancies per 1000 person-years. Women exposed to acne treatments with a label warning had 28.8 fewer pregnancies per 1000 person-years than the no-risk cohort. 
Table 1 Study sample characteristics

\begin{tabular}{|c|c|c|c|}
\hline Characteristics & $\begin{array}{l}\text { iPLEDGE } \\
(N=84,204)\end{array}$ & $\begin{array}{l}\text { Label warning } \\
(N=473,167)\end{array}$ & $\begin{array}{l}\text { No known risk } \\
(N=422,318)\end{array}$ \\
\hline \multicolumn{4}{|l|}{ Age group (years) } \\
\hline $15-19$ & $40,726(48.4)$ & $201,372(42.6)$ & $185,729(44.0)$ \\
\hline $20-29$ & $25,469(30.2)$ & $138,952(29.4)$ & $120,777(28.6)$ \\
\hline $30-39$ & $13,216(15.7)$ & $94,155(19.9)$ & $84,159(19.9)$ \\
\hline $40-44$ & $4793(5.7)$ & $38,688(8.2)$ & $31,653(7.5)$ \\
\hline Depression & $5732(6.8)$ & $35,132(7.4)$ & $32,872(7.8)$ \\
\hline \multicolumn{4}{|l|}{ Charlson Comorbidity Index } \\
\hline 0 & $80,026(95.0)$ & $445,023(94.1)$ & $393,304(93.1)$ \\
\hline 1 & $3531(4.2)$ & $23,683(5.0)$ & $24,108(5.7)$ \\
\hline$\geq 2$ & $647(0.8)$ & $4461(0.9)$ & $4906(1.2)$ \\
\hline History of contraceptive use & $28,716(34.1)$ & $100,361(21.2)$ & $97,073(23.0)$ \\
\hline History of pregnancy & $354(0.4)$ & $4401(0.9)$ & $6584(1.6)$ \\
\hline History of acne procedure & $6004(7.1)$ & $22,288(4.7)$ & $20,201(4.8)$ \\
\hline History of prescription acne treatment & $31,952(37.9)$ & $152,147(32.2)$ & $211,360(50.0)$ \\
\hline \multicolumn{4}{|l|}{ Health insurance type } \\
\hline Preferred provider organization & $52,845(62.8)$ & $294,739(62.3)$ & $255,896(60.6)$ \\
\hline \multicolumn{4}{|l|}{ Health insurance holder } \\
\hline Employee & $22,518(26.7)$ & $142,720(30.2)$ & $124,892(29.6)$ \\
\hline Spouse & $8544(10.1)$ & $62,649(13.2)$ & $56,399(13.4)$ \\
\hline Child & $53,142(63.1)$ & $267,798(56.6)$ & $241,027(57.1)$ \\
\hline \multicolumn{4}{|l|}{ Year of exposure } \\
\hline 2006 & $5298(6.3)$ & $28,199(6.0)$ & $25,260(6.0)$ \\
\hline 2007 & $6677(7.9)$ & $34,166(7.2)$ & $31,242(7.4)$ \\
\hline 2008 & $10,829(12.9)$ & $47,104(10.0)$ & $35,484(8.4)$ \\
\hline 2009 & $12,185(14.5)$ & $60,324(12.7)$ & $43,939(10.4)$ \\
\hline 2010 & $10,918(13.0)$ & $59,645(12.6)$ & $47,741(11.3)$ \\
\hline 2011 & $10,180(12.1)$ & $66,228(14.0)$ & $63,801(15.1)$ \\
\hline 2012 & $10,116(12.0)$ & $66,569(14.1)$ & $65,047(15.4)$ \\
\hline 2013 & $10,620(12.6)$ & $56,454(11.9)$ & $53,134(12.6)$ \\
\hline 2014 & $7298(8.7)$ & $49,872(10.5)$ & $49,898(11.8)$ \\
\hline 2015 & $83(0.1)$ & $4606(1.0)$ & $6772(1.6)$ \\
\hline \multicolumn{4}{|l|}{ Region of residence (USA) } \\
\hline Northeast & $11,190(13.3)$ & $75,964(16.1)$ & $89,940(21.3)$ \\
\hline North Central & $18,784(22.3)$ & $109,034(23.0)$ & $98,362(23.3)$ \\
\hline South & $35,451(42.1)$ & $193,841(41.0)$ & $142,486(33.7)$ \\
\hline West & 17,597 (20.9) & 88,157 (18.6) & $85,788(20.3)$ \\
\hline Unknown & $1182(1.4)$ & $6171(1.3)$ & $5742(1.4)$ \\
\hline
\end{tabular}

Data are presented as $N(\%)$ unless otherwise indicated
Stratification by age showed more attenuated differences among treatment groups for teenagers than women in their twenties or thirties (Table 4). Teenage girls who initiated isotretinoin had 2.9 (95\% CI 3.8 to 2.1) fewer pregnancies per 1000 person-years than same-age initiators of doxycycline/minocycline, reflecting a $63 \%$ decrease in fetal exposure risk. Compared with teenagers in the no-risk cohort, isotretinoin users had 3.7 (95\% CI 5 to 2.5) fewer pregnancies per 1000 person-years. See Table 1 in the ESM for more sensitivity analyses.

\section{Discussion}

In this population-based observational study, we found that the pregnancy risk during isotretinoin treatment did not approximate zero and resulted in a substantial proportion of abortions. Women who used isotretinoin had about 19 fewer pregnancies per 1000 person-years than women who used doxycycline or minocycline, whereas the latter had about 29 fewer pregnancies per 1000 person-years than women who used acne treatments with no fetal risk. Both absolute and 
Table 2 Contraceptive use during acne treatment

\begin{tabular}{llll}
\hline Contraceptive use & iPLEDGE & Label warning & No known risk \\
\hline $\begin{array}{l}\text { Overall contracep- } \\
\text { tive use }\end{array}$ & $41,734(49.5)$ & $118,695(25.1)$ & $96,567(22.9)$ \\
Prevalence of contraceptive use by age group, years \\
15-19 & 45 & 20 & 17 \\
$20-29$ & 62 & 38 & 36 \\
$30-39$ & 41 & 21 & 21 \\
$40-44$ & 23 & 13 & 12 \\
Types of contraceptives among contraceptive users \\
Oral contraceptive & $38,389(91.9)$ & $105,728(89.1)$ & $86,480(89.5)$ \\
Intrauterine device & $345(0.8)$ & $1506(1.3)$ & $961(1.0)$ \\
Implant & $102(0.2)$ & $408(0.3)$ & $233(0.2)$ \\
Injection & $1263(3.0)$ & $4714(4.0)$ & $3564(3.8)$ \\
Vaginal ring & $1354(3.2)$ & $5440(4.6)$ & $4534(4.7)$ \\
Patch & $281(0.7)$ & $899(0.8)$ & $795(0.8)$ \\
\hline
\end{tabular}

Data are presented as \% or $N(\%)$ unless otherwise indicated relative reductions of pregnancy rates among isotretinoin users were attenuated among teenagers, who constituted about $43-48 \%$ of acne treatment users in our data.

Our study is the first to report pregnancy rates during various acne treatments by women with demonstrated similar ages and clinical characteristics. The estimated pregnancy rate among clindamycin and erythromycin users was about 57 per 1000 users annually, which approximates national pregnancy rates [25]. Although doxycycline and minocycline users used contraceptives to an extent similar to that of clindamycin and erythromycin users, they showed about half the rate of fetal exposure. This finding may help quantify the impact of knowledge of teratogenic risk (in the absence of risk mitigation strategies) on pregnancy rates, which could be realized through a channeling effect. Specifically, women with a desire to become pregnant may have chosen or been directed to safer options. While intuitive, we did find similar utilization rates of hormonal contraceptives in these groups,
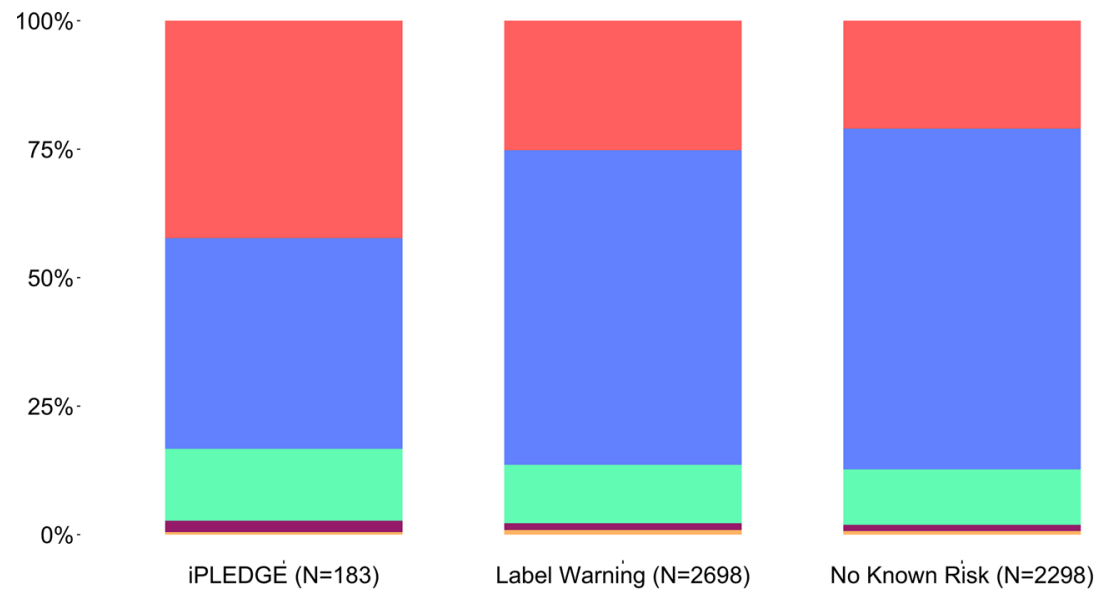

Abortion Live Birth Pregnancy (Unknown Outcome) Ectopic Pregnancy Stillbirth

Label Warning $(\mathrm{N}=2698)$

No Known Risk (N=2298)

\section{Risk Mitigation Strategy}

Fig. 1 Distribution of pregnancy outcomes among conceptions during acne treatment exposure

Table 3 Sample size, follow-up, number of events and incidence rates

\begin{tabular}{llll}
\hline & iPLEDGE & Label warning & No known risk \\
\hline Study sample & 84,204 & 473,176 & 422,318 \\
Follow-up time (days), median (IQR) & $172(100-204)$ & $30(30-98)$ & $30(20-30)$ \\
Pregnancies (count) & 183 & 2698 & 2298 \\
Person-years & 36,220 & 107,056 & 39,905 \\
Incidence rate per 1000 person-years & $5.0(4.3-5.8)$ & $25.2(24.3-26.2)$ & $57.6(55.3-60.0)$ \\
Incidence by age (years) & & & \\
$15-19$ & $1.7(1.2-2.4)$ & $4.6(4.1-5.2)$ & $5.5(4.5-6.7)$ \\
$20-29$ & $9.4(7.7-11.5)$ & $50.9(48.3-53.7)$ & $95.9(90.3-102)$ \\
$30-39$ & $8.5(6.4-11.4)$ & $55.2(51.9-58.8)$ & $142.5(134.3-151.3)$ \\
$40-44$ & $3.7(1.8-7.8)$ & $9.0(7.2-11.4)$ & $16.1(12.1-21.4)$ \\
\hline
\end{tabular}

Data in parentheses are $95 \%$ confidence intervals unless otherwise indicated $I Q R$ interquartile range 
Table 4 Relative and absolute pregnancy risk comparison among exposure groups after adjustment with stabilized inverse probability of treatment weights

\begin{tabular}{llll}
\hline & iPLEDGE vs. label warning & iPLEDGE vs. no known risk & Label warning vs. no known risk $^{\mathrm{b}}$ \\
\hline $\begin{array}{l}\text { Crude estimates } \\
\quad \text { Incidence rate difference }\end{array}$ & $-20.1(-21.3$ to -18.9$)$ & $-52.5(-55.0$ to -50.1$)$ & $-32.4(-34.9$ to -29.8$)$ \\
$\quad$ Incidence rate ratio & $0.20(0.17-0.23)$ & $0.09(0.07-0.10)$ & $0.43(0.41-0.46)$ \\
SIPTW estimates & & & $-28.8(-31.2$ to -26.3$)$ \\
$\quad$ Incidence rate difference & $-19.1(-20.3$ to -17.9$)$ & $-50.1(-52.6$ to -47.6$)$ & $0.47(0.44-0.50)$ \\
Incidence rate ratio & $0.23(0.20-0.26)$ & $0.11(0.09-0.12)$ & $-0.5(-1.7$ to 0.7$)$ \\
Age (years) stratification of SIPTW incidence rate difference & & $-3.7(-5.0$ to -2.5$)$ & $-41.3(-47.6$ to -35.0$)$ \\
15-19 & $-2.9(-3.8$ to -2.1$)$ & $-83.5(-89.6$ to -77.5$)$ & $-78.1(-87.1$ to -69.1$)$ \\
$20-29$ & $-40.2(-43.5$ to -37.0$)$ & $-130.0(-139.0$ to -121.0$)$ & $-6.2(-11.2$ to -1.2$)$ \\
$30-39$ & $-45.9(-50.2$ to -41.6$)$ & $-11.0(-16.5$ to -5.5$)$ & \\
$40-44$ & $-4.5(-8.2$ to -0.8$)$ & &
\end{tabular}

Figures in parentheses are $95 \%$ confidence intervals

SIPTW stabilized inverse probability treatment weighting, WCBP Women of childbearing potential,

'The 'label warning' cohort was the reference group

bThe 'no known risk' cohort was the reference group

${ }^{c}$ Estimates are per 1000 person-years

though the overall low utilization rates leave room for differences in reliance on other contraceptive methods, which we could not measure in our database.

The fetal exposure rate to isotretinoin in our study is consistent with previous data from Kaiser Permanente Southern and Northern California (5.8/1000 person-years; 11 events were found among 3844 users assuming 6 months of treatment duration) during the first 2 years of iPLEDGE implementation [4]. Our data suggest consistent fetal exposure rates in more recent years among a similar population of privately insured women. Because iPLEDGE requires every woman of childbearing age to commit to contraception or abstinence during isotretinoin treatment, we can safely assume that nearly all pregnancies during isotretinoin treatment are unintended. In contrast, topical clindamycin or erythromycin users in particular would be expected to include a disproportionate number of women with a desire to become pregnant, which is supported by the lower frequencies of contraceptive use. Consequently, the observed lower fetal exposure rate among isotretinoin users cannot be solely attributed to the effectiveness of iPLEDGE but likely represents a mixture of the REMS, general knowledge about the teratogenic risk, and channeling of women with pregnancy desires to safer options, which is not measurable in our claims data. The observed differences in fetal exposure rates between doxycycline/minocycline and our no-risk cohort provide some context for the potential effect of such channeling, although this effect might be even stronger considering the more pronounced fetal risk of isotretinoin.

Channeling women who are planning pregnancy away from isotretinoin should be less relevant among teenagers, who represent nearly half of the patients with acne in our data and for whom the vast majority of pregnancies are unintentional [6]. Indeed, although pregnancy rates were low, differences between exposure groups were less pronounced, which could be attributed to less channeling or reduced iPLEDGE effectiveness in this age group.

Reported key causes of iPLEDGE failure include noncompliance with and ineffectiveness of contraceptives [26-28]. Reports from other countries that require or recommend the use of contraceptives for WCBPs who use isotretinoin have reported a similar prevalence of contraceptive use or lower [29-31]. Moreover, we found that about $90 \%$ of contraceptive users in our study population preferred oral forms, and only $1 \%$ used more effective long-acting reversible contraceptives, which is consistent with national estimates [32, 33] although not recommended [34, 35]. Thus, a focus on the selection of effective contraceptives and their consistent use, even with limited sexual activity, is important when advising patients who initiate teratogenic drugs.

Our data indicated that only $45 \%$ of teenagers who used isotretinoin used contraceptives, compared with $62 \%$ of adults in their twenties. In a survey-based study [27], about $19 \%$ of women who had indicated abstinence instead of using hormonal contraceptives reported sexual activity during isotretinoin treatment. In the same data, isotretinoin users who chose abstinence were significantly younger than women who used hormonal contraceptives. Reports suggest that few teenagers $(<20 \%)$ are willing to seek healthcare related to birth control if their parents are involved in these healthcare encounters [36], suggesting that sexually active teenagers may indicate abstinence when discussing contraception requirements. 
Healthcare professionals considering initiating isotretinoin therapy in teenagers must consider potential problems accessing contraceptives, uncertainty about the need for contraception during the initial phase of becoming sexually active, and increased risk-taking behavior. Future research should evaluate patients and other contextual characteristics that may affect the effectiveness of REMS to allow the development of targeted strategies to optimize cost (burden) benefit.

Strengths of our study include a large population-based sample that allowed age-specific comparisons and use of validated methods with high positive predictive value to detect pregnancies and estimate conception. Some potential confounders, including acne severity, sexual activity, and desire for pregnancy, were not directly measurable. We attempted to indirectly adjust for acne severity by capturing previous acne treatments and for sexual activity based on obstetric history, but residual imbalances are possible, especially considering channeling issues related to pregnancy desire discussed earlier. Furthermore, factors such as low income or lack of health insurance are associated with higher rates of unintended pregnancy [37], which means our results are not generalizable beyond privately insured women. Because we required only 6 months as the look-back period, longacting reversible contraceptives that may have been inserted or implanted several years earlier may have been missed. Finally, while we had generally small error margins in the range of 1-2 weeks $[13,15]$, gestational age and therefore conception was estimated, and some assumed fetal exposures may have been misclassified.

\section{Conclusion}

Fetal exposure to acne treatments varied according to levels of teratogenicity. Women who used isotretinoin had about one-quarter the fetal exposure rates of women exposed to doxycycline or minocycline, who in turn showed about half the pregnancy rates of women who used acne treatment without teratogenic risks. Teenagers had generally low pregnancy rates though less pronounced differences across acne treatments. Further research is needed to understand factors that may alter the effectiveness of REMS to design tailored risk mitigation approaches that reduce the number of unintended pregnancies while minimizing unnecessary burden on patients and healthcare providers.

Supplementary Information The online version contains supplementary material available at https://doi.org/10.1007/s40264-021-01053-3.

\section{Declarations}

Funding No sources of funding were used to conduct this study or prepare this manuscript.
Conflict of interest Yasser Albogami, Amir Sarayani, Juan HincapieCastillo, and Almut G. Winterstein have no conflicts of interest that are directly relevant to the content of this article.

Ethics approval The study was exempted from approval by the Institutional Review Board at the University of Florida because deidentified data were used.

Consent to participate Not applicable.

Consent for publication All authors read the final version of the manuscript and gave consent for publication.

Availability of data and material Data may be obtained from a third party and are not publicly available.

Code availability Available upon request.

Author contributions All authors were involved in the conception and design, interpretation of data, drafting the manuscript or revising it critically for important intellectual content.

Open Access This article is licensed under a Creative Commons Attribution-NonCommercial 4.0 International License, which permits any non-commercial use, sharing, adaptation, distribution and reproduction in any medium or format, as long as you give appropriate credit to the original author(s) and the source, provide a link to the Creative Commons licence, and indicate if changes were made. The images or other third party material in this article are included in the article's Creative Commons licence, unless indicated otherwise in a credit line to the material. If material is not included in the article's Creative Commons licence and your intended use is not permitted by statutory regulation or exceeds the permitted use, you will need to obtain permission directly from the copyright holder. To view a copy of this licence, visit http://creativecommons.org/licenses/by-nc/4.0/.

\section{References}

1. Kovitwanichkanont T, Driscoll T. A comparative review of the isotretinoin pregnancy risk management programs across four continents. Int J Dermatol. 2018;57:1035-46. https://doi. org/10.1111/ijd.13950.

2. Lammer EJ, Chen DT, Hoar RM, Agnish ND, Benke PJ, Braun JT, et al. Retinoic acid embryopathy. N Engl J Med. 1985;313:83741. https://doi.org/10.1056/NEJM198510033131401.

3. Abroms L, Maibach E, Lyon-Daniel K, Feldman SR. What is the best approach to reducing birth defects associated with isotretinoin? PLoS Med. 2006;3. Available from: https://www.ncbi.nlm. nih.gov/pmc/articles/PMC1637125/

4. Shin J, Cheetham TC, Wong L, Niu F, Kass E, Yoshinaga MA, et al. The impact of the iPLEDGE program on isotretinoin fetal exposure in an integrated health care system. J Am Acad Dermatol. 2011;65:1117-25. Available from: https://www.jaad.org/artic le/S0190-9622(10)01101-1/abstract

5. Barbieri JS, Frieden IJ, Nagler AR. Isotretinoin, Patient Safety, and Patient-Centered Care-Time to Reform iPLEDGE. JAMA Dermatol [Internet]. American Medical Association; 2020 [cited 2020 Apr 20];156:21-2. Available from: https://jamanetwor k.com/journals/jamadermatology/fullarticle/2753270

6. Finer LB, Zolna MR. Declines in Unintended Pregnancy in the United States, 2008-2011 [Internet]. https://doi.org/10.1056/ NEJMsa1506575. Massachusetts Medical Society; 2016 [cited 2020 Apr 20]. Available from: https://www.nejm.org/doi/10.1056/ 
NEJMsa1506575?url_ver=Z39.88-2003\&rfr_id=ori\%3Arid \%3Acrossref.org\&rfr_dat $=$ cr_pub++0www.ncbi.nlm.nih.gov

7. Ejaz A, Malaiyandi V, Kim WB, Rogalska T, Alhusayen R. Validating the diagnostic code for acne in a tertiary care dermatology centre. Eur J Dermatol EJD. 2015;25:469-71.

8. Adam MP, Polifka JE, Friedman JM. Evolving knowledge of the teratogenicity of medications in human pregnancy. Am $\mathrm{J}$ Med Genet C Semin Med Genet. 2011;157:175-82. https://doi. org/10.1002/ajmg.c.30313.

9. SOLODYN ${ }^{\circledR}$ HIGHLIGHTS OF PRESCRIBING INFORMATION [Internet]. [cited 2020 Apr 19]. Available from: https:// accessdata.fda.gov/drugsatfda_docs/label/2011/050808s014lbl. pdf.

10. DOXTERIC HIGHLIGHTS OF PRESCRIBING INFORMATION [Internet]. [cited 2020 Apr 19]. Available from: https:// www.accessdata.fda.gov/drugsatfda_docs/label/2014/050795Orig 1s019lbl.pdf.

11. MotherToBaby. (2020). The Baby Blog. Retrieved from [Internet]. 2020. Available from: https://mothertobaby.org/baby-blog/.

12. RISK EVALUATION AND MITIGATION STRATEGY (REMS): The iPLEDGE Program [Internet]. 2012 [cited 2020 Apr 20]. Available from: https://www.fda.gov/downloads/Drugs/DrugS afety/PostmarketDrugSafetyInformationforPatientsandProviders/ UCM234639.pdf.

13. Matcho A, Ryan P, Fife D, Gifkins D, Knoll C, Friedman A. Inferring pregnancy episodes and outcomes within a network of observational databases. PLoS ONE. 2018;13. Available from: https:// www.ncbi.nlm.nih.gov/pmc/articles/PMC5794136/.

14. Zhu Y, Hampp C, Wang X, Albogami Y, Wei Y-JJ, Brumback $\mathrm{BA}$, et al. Validation of algorithms to estimate gestational age at birth in the Medicaid Analytic eXtract-quantifying the misclassification of maternal drug exposure during pregnancy. Pharmacoepidemiol Drug Saf. 2020;29:1414-22. https://doi.org/10.1002/ pds.5126.

15. Hornbrook MC, Whitlock EP, Berg CJ, Callaghan WM, Bachman DJ, Gold R, et al. Development of an algorithm to identify pregnancy episodes in an integrated health care delivery system. Health Serv Res [Internet]. 2007 [cited 2020 Apr 20];42:908-27. Available from: https://www.ncbi.nlm.nih.gov/pmc/articles/ PMC1955367/.

16. Sarayani A, Albogami Y, Elkhider M, Hincapie-Castillo JM, Brumback BA, Winterstein AG. Comparative effectiveness of risk mitigation strategies to prevent fetal exposure to mycophenolate. BMJ Qual Saf [Internet]. BMJ Publishing Group Ltd; 2019 [cited 2020 Apr 20]; Available from: https://qualitysafety.bmj.com/conte nt/early/2019/10/24/bmjqs-2019-010098.

17. DEPO-PROVERA HIGHLIGHTS OF PRESCRIBING INFORMATION [Internet]. [cited 2020 May 6]. Available from: https:// www.accessdata.fda.gov/drugsatfda_docs/label/2010/020246s036 lbl.pdf.

18. Lim HW, Collins SAB, Resneck JS, Bolognia JL, Hodge JA, Rohrer TA, et al. Contribution of health care factors to the burden of skin disease in the United States. J Am Acad Dermatol. 2017;76(1151-1160):e21.

19. Charlson ME, Pompei P, Ales KL, MacKenzie CR. A new method of classifying prognostic comorbidity in longitudinal studies: development and validation. J Chronic Dis. 1987;40:373-83.

20. Fiest KM, Jette N, Quan H, St. Germaine-Smith C, Metcalfe A, Patten SB, et al. Systematic review and assessment of validated case definitions for depression in administrative data. BMC Psychiatry. 2014;14:289. https://doi.org/10.1186/s12888-014-0289-5.

21. Haddad LB, Monsour M, Tepper NK, Whiteman MK, Kourtis AP, Jamieson DJ. Trends in contraceptive use according to HIV status among privately insured women in the United States. Am J Obstet Gynecol. 2017;217:676.e1-676.e11.
22. Snyder AH, Weisman CS, Liu G, Leslie D, Chuang CH. The impact of the affordable care act on contraceptive use and costs among privately insured women. Womens Health Issues Off Publ Jacobs Inst Womens Health. 2018;28:219-23.

23. Austin PC, Stuart EA. Moving towards best practice when using inverse probability of treatment weighting (IPTW) using the propensity score to estimate causal treatment effects in observational studies. Stat Med. 2015;34:3661-79.

24. Wickham H. ggplot2: Elegant Graphics for Data Analysis [Internet]. Springer-Verlag New York; 2016 [cited 2020 Apr 20]. Available from: https://ggplot2.tidyverse.org.

25. National Vital Statistics Reports, Volume 66, Number 1, January 5, 2017. p. 70 .

26. Choi JS, Koren G, Nulman I. Pregnancy and isotretinoin therapy. CMAJ Can Med Assoc J [Internet]. 2013 [cited 2020 Apr 22];185:411-3. Available from: https://www.ncbi.nlm.nih.gov/ pmc/articles/PMC3602257/.

27. Collins M-K, Moreau JF, Opel D, Swan J, Prevost N, Hastings $\mathrm{M}$, et al. Compliance with pregnancy prevention measures during isotretinoin therapy. J Am Acad Dermatol [Internet]. 2014 [cited 2020 Apr 22];70:55-9. Available from: http://www.sciencedirect. com/science/article/pii/S0190962213009092.

28. Pinheiro SP, Kang EM, Kim CY, Governale LA, Zhou EH, Hammad TA. Concomitant use of isotretinoin and contraceptives before and after iPledge in the United States. Pharmacoepidemiol Drug Saf. 2013;22:1251-7. https://doi.org/10.1002/pds.3481.

29. Crijns HJMJ, Rein N van, Wied CCG, Straus SM, Berg LTW de $\mathrm{J}$ den. Prescriptive contraceptive use among isotretinoin users in the Netherlands in comparison with non-users: a drug utilisation study. Pharmacoepidemiol Drug Saf. 2012;21:1060-6. https://doi. org/10.1002/pds.3200.

30. Henry D, Dormuth C, Winquist B, Carney G, Bugden S, Teare G, et al. Occurrence of pregnancy and pregnancy outcomes during isotretinoin therapy. CMAJ Can Med Assoc J [Internet]. 2016 [cited 2020 Apr 22];188:723-30. Available from: https://www. ncbi.nlm.nih.gov/pmc/articles/PMC4938682/.

31. Uusküla A, Pisarev H, Kurvits K, Laius O, Laanpere M, Uusküla M. Compliance with Pregnancy Prevention Recommendations for Isotretinoin in Estonia in 2012-2016. Drugs - Real World Outcomes [Internet]. 2018 [cited 2020 Apr 22];5:129-36. Available from: https://www.ncbi.nlm.nih.gov/pmc/articles/PMC5984611/.

32. Daniels K, Dougherty J, Jones J. Current contraceptive status among women aged 15-44: United States, 2011-2013. U.S. Department of Health and Human Services, Centers for Disease Control and Prevention, National Center for Health Statistics; 2014.

33. Jones J, Mosher W, Daniels K. Current contraceptive use in the United States, 2006-2010, and changes in patterns of use since. Natl Health Stat Rep. 1995;2012:1-25.

34. Trussell J. Contraceptive failure in the United States. Contraception [Internet]. 2011 [cited 2020 Apr 22];83:397-404. Available from: https://www.ncbi.nlm.nih.gov/pmc/articles/PMC3638209/.

35. Winner B, Peipert JF, Zhao Q, Buckel C, Madden T, Allsworth $\mathrm{JE}$, et al. Effectiveness of long-acting reversible contraception. N Engl J Med. 2012;366:1998-2007. https://doi.org/10.1056/ NEJMoa1110855.

36. Marks A, Malizio J, Hoch J, Brody R, Fisher M. Assessment of health needs and willingness to utilize health care resources of adolescents in a suburban population. J Pediatr [Internet]. 1983 [cited 2020 Apr 22];102:456-60. Available from: http://www. sciencedirect.com/science/article/pii/S0022347683806775.

37. Cubbin C, Braveman PA, Marchi KS, Chavez GF, Santelli JS, Gilbert BJC. Socioeconomic and racial/ethnic disparities in unintended pregnancy among postpartum women in California. Matern Child Health J. 2002;6:237-46. 